\title{
Agrotóxicos e Transgênicos: Uma Crítica Popular
}

\author{
Rodrigo Ferraz Ramos ${ }^{1}$ \\ Antônio Inácio Andrioli ${ }^{2}$ \\ Débora Leitzke Betemps ${ }^{3}$
}

Resumo: Diversas críticas foram inferidas contra a revolução verde e sua variante denominada revolução duplamente verde. A primeira introduziu o uso de agrotóxicos e, a segunda, os transgênicos na agricultura. Apesar do movimento estudantil da agronomia corroborar com a discussão acerca da temática dos agrotóxicos e transgênicos, são escassos os trabalhos na literatura científica que abordem as contribuições do movimento estudantil nas discussões dessa temática. Por isso, objetivou-se abordar o debate acerca dos agrotóxicos e transgênicos na perspectiva do movimento estudantil nacional da agronomia. Realizou-se uma revisão bibliográfica acerca da temática e posteriormente, conduziu-se uma análise sobre $o$ posicionamento da militância da Federação de Estudantes de Agronomia do Brasil (FEAB) acerca do tema proposto. Observou-se que o movimento estudantil nacional da agronomia conduz diversas iniciativas em prol da ampliação da discussão acerca dos riscos do uso dos agrotóxicos e as incertezas do uso de transgênicos na agricultura. Conclui-se que as iniciativas do movimento estudantil nacional estão em defesa de uma agricultura sustentável.

Palavras-chave: Agricultura; Movimento Estudantil; Revolução Verde.

\begin{abstract}
Several criticisms were made against the green revolution and its so-called double green revolution. The first introduced the use of pesticides and, second, the transgenics in agriculture. Although the student movement of agronomy corroborates the discussion about the subject of pesticides and transgenics, there are few studies in the scientific literature that address the contributions of the student movement in the discussions of this topic. Therefore, the objective was to approach the debate about pesticides and transgenics in the perspective of the national student movement of agronomy. A bibliographical review on the subject was carried out and later, an analysis was carried out on the position of the militancy of the Federation of Students of Agronomy of Brazil (FEAB) about the proposed theme. It was observed that the national student movement of agronomy conducts several initiatives in order to broaden the discussion about the risks of the use of agrochemicals and the uncertainties of the use of transgenics in agriculture. It is concluded that the initiatives of the national student movement are in defense of a sustainable agriculture.
\end{abstract}

Keywords: Agriculture; Student Movement; Green Revolution.

\footnotetext{
1 Graduando em Agronomia, Universidade Federal da Fronteira Sul (UFFS), e-mail: rodrigoferrazramos@gmail.com

${ }^{2} \mathrm{PhD}$ em Sociologia pela Johannes Kepler Universitat Linz, docente e Vice-Reitor na Universidade Federal da Fronteira Sul (UFFS), e-mail: andrioli@uffs.edu.br

${ }^{3} \mathrm{PhD}$ em Agronomia pela UFPel, Professora Adjunto III na Universidade Federal da Fronteira Sul (UFFS), email: debora.betemps@uffs.edu.br
} 


\section{INTRODUÇÃO}

O processo de "Revolução Verde" foi baseado na difusão de tecnologias para o campo com intuito de "modernizar" a agricultura. A moto-mecanização, o uso de pesticidas e de variedades agrícolas altamente dependentes do uso de adubos solúveis constituíram o "pacote tecnológico" difundido por diversos profissionais das ciências agrárias (MACHADO; MACHADO FILHO, 2014). Apesar dos diversos problemas de ordem socioeconômica e ambiental advindos desse processo de desenvolvimento, e a não resolução do problema da fome no mundo (HARARI, 2016), uma "revolução duplamente verde", baseada no uso de sementes geneticamente modificadas por transgenia foi posteriormente difundida na agricultura (STANDAGE, 2010; ANDRIOLI; FUNCHS, 2012).

A discussão sobre a problemática do uso de agrotóxicos e as incertezas acerca do uso de transgênicos na agricultura são temas que surgem no âmago dos conflitos e correlações de força na sociedade, onde observa-se que diversos setores da sociedade incutiram críticas ao modelo de desenvolvimento da agricultura, desde pesquisadores a movimentos sociais do campo e da cidade, além de movimentos estudantis nacional (MOTTA, 2014; OTERO; LAPEGNA, 2016). No movimento estudantil das ciências agrárias, o movimento estudantil da agronomia representa uns dos principais segmentos que aglutinam a juventude para o debate em torno de temas que são de interesse dos estudantes e da sociedade em geral (RAMOS et al., 2017a).

O movimento estudantil nacional da agronomia é representado pela Federação de Estudantes de Agronomia do Brasil (FEAB). A FEAB é uma entidade estudantil que atua no território nacional articulando-se principalmente com os estudantes da agronomia nas diferentes universidades que abrangem o País. Um dos principais objetivos da FEAB é articular nacionalmente os estudantes da agronomia com o intuito de abordar reflexões que permeiam a formação profissional (RAMOS et al., 2017a). Entre os temas comumente abordados pelo conjunto da federação em seus espaços de atuação, destaca-se a temática dos agrotóxicos e transgênicos (FEAB, 2014).

Apesar do movimento estudantil nacional da agronomia compor os segmentos sociais que corroboram com a discussão da temática dos agrotóxicos e transgênicos, são escassos os

Revista Extensão em Foco, nº 17, Out./ Dez. (2018), p. 40 - 53. 
trabalhos na literatura científica que abordam as contribuições do movimento estudantil nas discussões de temas que são de interesse dos estudantes e da sociedade em geral.

Diante do exposto, objetiva-se realizar uma breve contextualização acerca da temática dos agrotóxicos e transgênicos e, em sequência, realizar uma síntese do debate suscitado pelo movimento estudantil nacional da agronomia sobre a referida temática, com destaque para as principais iniciativas, referências e posicionamentos empregados pela FEAB em relação ao tema proposto.

\section{MATERIAIS E MÉTODOS}

No presente estudo uma revisão da literatura especializada acerca da temática dos agrotóxicos e transgênicos foi realizada durante os meses de Outubro de 2017 a Abril de 2018. Consultou-se livros acerca do tema disponíveis na Biblioteca da Universidade Federal da Fronteira Sul (UFFS) e realizou-se uma busca de artigos em periódicos nacionais e internacionais. As buscas foram realizadas no Google Acadêmico e nas bases de dados da Scientific Electronic Library Online (SciELO), Embase e Citation Indexes. Realizou-se a busca por termos livres, sem o uso de vocabulário controlado.

Concomitantemente, foram analisados sistematicamente as cartilhas e demais arquivos bibliográficos da Federação de Estudantes de Agronomia do Brasil (FEAB) disponíveis eletronicamente no site da entidade (feab.wordpress.com) e que abordassem a temática dos agrotóxicos e transgênicos.

A partir da revisão da literatura especializada e da análise dos materiais bibliográficos da FEAB, em um primeiro momento, foi realizado uma introdução ao panorama do uso de agrotóxicos e transgênicos no contexto do desenvolvimento da agricultura, apresentando os principais discursos sociopolíticos que envolvem a temática. Posteriormente, conduziu-se uma discussão da inserção e a contribuição do movimento estudantil da agronomia no debate, com a descrição das principais iniciativas da militância e seus referenciais teóricos comumente adotados como subsídio nos debates e na elaboração de cartilhas e demais materiais.

\section{RESULTADOS E DISCUSÃO}

\section{Panorama do uso de agrotóxicos e transgênicos}

Revista Extensão em Foco, nº 17, Out./ Dez. (2018), p. 40 - 53. 
No Brasil, o processo de desenvolvimento da agricultura conduzido a partir da década de 1960 ficou conhecida como "Revolução Verde". Esse processo de desenvolvimento popularizou-se por ter contribuído para o aumento da produção agrícola por área cultivada e a produção mundial de commodities agrícolas (PINTOR; SILVA; PIACENTI, 2015).

Para Mazoyer e Roudart (2010), a denominada "Revolução Verde" não se caracteriza enquanto uma revolução agrícola per si, mas sim como uma variante da revolução agrícola dos tempos modernos. A base desse modelo de desenvolvimento da agricultura é a seleção de cultivares agrícolas com alto rendimento potencial e a seleção genética de animais domésticos com alto potencial de produção; utilização de motorização e mecanização das lavouras; alta dependência de pesticidas agrícolas e de fertilizantes químicos solúveis; ampla difusão no uso de sistemas de irrigação e drenagem, bem como, dependência de políticas de incentivos econômicos e fiscais para a promoção da agricultura contemporânea (KNICKEL et al., 2017; SIMTION, 2017).

Contudo, tal processo igualmente promoveu diversos problemas socioeconômicos e ambientais (FISCHER et al., 2015; BONNY, 2016). Na perspectiva ambiental, observou-se a presença e a contaminação de solos e de águas superficiais e subterrâneas por pesticidas, nitrato e outros contaminantes emergentes oriundos de atividades agrícolas (GOMES; ARIZON, 2014; LLORCA et al., 2016), com potencial para provocar distúrbios e desequilíbrios ambientais nas comunidades de organismos aquáticos (KHOSHNOOD, 2017; LI et al., 2017), declínio de populações de polinizadores (LIMA; ROCHA, 2012; AMULEN et al., 2017) e contaminação de agricultores (SANTANA; MOURA; NOGUEIRA, 2013; BURNS; PASTOOR, 2017).

Na perspectiva socioeconômica, como observado por Mazoyer e Roudart (2010), o aumento da produção de commodities promoveu uma oferta excessiva no mercado mundial, provocando consequentemente uma queda geral dos preços, levando a um estado de crise na agricultura. Assim, observou-se um processo de "desenvolvimento-e-crise", onde a capitalização e desenvolvimento de alguns tipos de agricultores foi acompanhado por processos de descapitalização e crise de outros (SILVA NETO; BASSO, 2005). Esse fenômeno promoveu um aumento da pobreza de agricultores menos capitalizados e consequente aumento no êxodo rural, com forte migração da população rural para as periferias das cidades (FONTOURA, 2016).

Revista Extensão em Foco, nº 17, Out./ Dez. (2018), p. 40 - 53. 
Os resultados desse processo reforçam a premissa de que um maior crescimento econômico em concomitância com um maior conhecimento científico e com a difusão de tecnologias sofisticadas não significam necessariamente a melhoria das condições de vida das populações mais vulnerabilizadas (PORTO; SOARES, 2012). Corroborando com essa asserção, observou-se que mesmo após a difusão da Revolução Verde e suas tecnologias para o campo, das cerca de três bilhões de pessoas que vivem em áreas rurais, aproximadamente 800 milhões passam fome (BELIK; CORREA, 2013), onde estima-se que ainda em 2030 a pobreza extrema nas áreas rurais será de 7,5\% da população rural (LABORD; MARTIN, 2016).

Standage (2010), destaca que a resposta a longo prazo para essa crise alimentar seria uma "segunda revolução verde" ou uma "revolução duplamente verde". Para o mesmo autor, alguns defensores dessa segunda revolução enfatizam o potencial de sementes geneticamente modificadas, que produzem seus próprios pesticidas ou são projetadas para fazer um uso mais eficiente de água e fertilizantes. (STANDAGE, 2010)

No Brasil, o cultivo de plantas geneticamente modificadas pela técnica da transgenia iniciou-se no final da década de 1990 com a soja transgênica, cultivada ilegalmente no Estado do Rio Grande do Sul, sendo que somente no ano de 2005 regulamentou-se legalmente o plantio e comercialização de variedades transgênicas no país (RIBEIRO; MARIN, 2012).

Entre as principais vantagens propagandeadas para o uso de cultivares transgênicas, destacaram-se as seguintes: aumento da produção e da produtividade com redução de custos; melhor controle ambiental, especialmente pela redução ou extinção do uso de agrotóxicos; plena abertura de oportunidades para evitar o aparecimento de monopólios ou oligopólios na produção de sementes melhoradas; consistente alternativa para contribuir com a mitigação ou extinção da fome e da pobreza, entre outros (BARROWS; SEXTON; ZILBERMAN, 2014; KLÜMPER; QAIM, 2014).

Para Andrioli e Funchs (2012), os agricultores são convencidos a utilizar sementes transgênicas pelos mesmos argumentos propagados na época da introdução dos agrotóxicos na agricultura e tendem a ser eliminados do processo produtivo em função dos crescentes custos de produção. Contudo, Klümper e Qaim (2014) em uma meta-análise demonstram que a adoção de organismos geneticamente modificados na agricultura proporcionou, em média, um aumento no rendimento das colheitas de $22 \%$ e reduziu o uso de agrotóxicos em $37 \%$.

Revista Extensão em Foco, nº 17, Out./ Dez. (2018), p. 40 - 53. 
Outros estudos, como de Almeida et al. (2017), observaram que para cultura da soja, durante o período de 2000 a 2012 o aumento de um ponto percentual (pp) na produtividade demandou o aumento de 13 pp no uso de agrotóxicos em geral, em especial ao uso de herbicidas por unidade de área, que cresceu $64 \%$, podendo significar um aumento no uso e nos gastos com agrotóxicos.

Os principais responsáveis pela difusão dos agrotóxicos e transgênicos, ou seja, das tecnologias consideradas "modernas" para a agricultura, foram os profissionais da ciência agronômica (SILVA NETO, 2009; SILVA 2011; MACHADO; MACHADO FILHO, 2014). Assim, a temática dos agrotóxicos e transgênicos ganha especial importância para o segmento estudantil, devido ao fato do modelo de formação profissional da agronomia ser um dos principais temas abordados pelo conjunto do movimento estudantil nacional da agronomia (RAMOS et al., 2017a).

\section{A crítica do movimento estudantil}

Durante o processo de "Revolução Verde" e da "Revolução Duplamente Verde" os profissionais da ciência agronômica contribuíram para o processo de desenvolvimento desigual no meio rural, principalmente por meio da atuação extensionista desses profissionais que desempenharam papel fundamental na difusão e aplicação das tecnologias comumente designadas "modernas" na agricultura. Esse processo ocorreu, em partes, devido ao fato que hegemonicamente a ciência agronômica constitui uma disciplina cuja problemática estaria centrada no rendimento físico das plantas e dos animais (SILVA NETO, 2010).

Freire (1983), já havia observado que o papel do agrônomo extensionista não poderia se limitar meramente a esfera da substituição dos procedimentos empíricos dos agricultores por técnicas e tecnologias consideradas "modernas". De fato, diversas críticas foram incutidas aos profissionais da ciência agronômica por pesquisadores, movimentos sociais, movimentos estudantis e sociedade em geral, no qual culminaram em diversas iniciativas que objetivavam denunciar os problemas oriundos da Revolução Verde e propor alternativas de desenvolvimento sustentável para meio rural (RAMOS et al., 2017b; SILVA NETO, 2017).

No movimento estudantil nacional, a Federação de Estudantes de Agronomia do Brasil (FEAB) representa uns dos principais segmentos que conglobam esforços na tentativa de propor

Revista Extensão em Foco, nº 17, Out./ Dez. (2018), p. 40 - 53. 
e defender alternativas de desenvolvimento sustentável (VIEIRA; SOUZA; SOUZA, 2016). A FEAB aborda temas e reflexões que permeiam a formação profissional da agronomia, sendo que, entre principais temas comumente abordados pelo conjunto da federação em seus espaços de atuação, destaca-se a temática dos agrotóxicos e transgênicos (FEAB, 2014).

A FEAB é uma entidade estudantil que objetiva sensibilizar os estudantes para práticas de intervenção que tenha o objetivo de transformação social (VIEIRA; SOUZA; SOUZA, 2016). Atua no território nacional articulando-se principalmente com os estudantes da agronomia e ao longo de sua história foi protagonista e apoiadora de diversas iniciativas populares e caráter progressista, como salienta Ramos et al. (2017a, p. 138):

Dentre as principais contribuições da FEAB, destacam-se o apoio ao fim da Lei do Boi, que destinava uma cota de $50 \%$ de vagas nas Universidades para filhos de fazendeiros, a criação do Currículo Mínimo Nacional, a Lei dos Agrotóxicos (receituário agronômico) e a ampliação das discussões sobre a necessidade de modelos agrícolas alternativos ao da "Revolução Verde".

No Brasil, diversas universidades possuem núcleos de estudantes que se organizam no movimento estudantil nacional da agronomia. Cada grupo possui autonomia própria em nível local e o método empregado pela federação para a discussão de temas que são de interesse dos estudantes é semelhante ao método adotado pelos grupos de estudos em agroecologia, onde ocorre a realização de encontros presenciais semanalmente ou quinzenalmente, realizando-se a leitura prévia dos materiais bibliográficos escolhidos pelos membros do grupo, e posterior discussão sistematiza pelos membros (DEMARCHI et al., 2011; RAMOS et al., 2017b).

Nos espaços de discussão da federação o tema dos agrotóxicos e transgênicos são amplamente abordados. Durante as reuniões da militância algumas referências são comumente abordadas e discutidas. Entre aquelas que discutem os riscos dos agrotóxicos, destacam-se as seguintes obras: “A Agricultura Ecológica e a Máfia dos Agrotóxicos no Brasil” (PINHEIRO; NASR; LUZ, 1993), que relata diversos problemas oriundos do uso abusivo de agrotóxicos e concomitantemente aborda a agricultura ecológica enquanto uma alternativa sustentável para a agricultura; "Plantas Doentes pelo Uso de Agrotóxicos", de Francis Chaboussou (2006), que discorre sobre a hipótese de que plantas equilibradas nutricionalmente não são atacadas por parasitas, dispensando-se o uso de agrotóxicos.

Revista Extensão em Foco, nº 17, Out./ Dez. (2018), p. 40 - 53. 
Entre as referências discutidas pela militância que discorrem sobre os riscos e incertezas dos transgênicos na agricultura, destacam-se as seguintes obras: "Transgênicos para quem?", de Zanoni e Ferment (2011), que abordam desde problemas sociais até problemas ambientais e os potenciais riscos a biodiversidade promovidos pelo uso de transgênicos na agricultura e; "Transgênicos: As Sementes do Mal”, de Andrioli e Fuchs (2012), que discorre sobre as incertezas e os potenciais perigos do uso de transgênicos, bem como, versa sobre a hegemonia das multinacionais do setor do agronegócio.

A FEAB também discute materiais elaborados pelo próprio movimento estudantil da agronomia, como por exemplo, o "Livro Cinza do Agronegócio" (FEAB, 2010), que discorre sobre a Revolução Verde na perspectiva do processo de desenvolvimento da agricultura dos tempos modernos, bem como aborda o lobby das empresas privadas para liberação dos agrotóxicos e transgênicos e as implicações da difusão e uso desse "pacote tecnológico" na agricultura. Assim, para a FEAB (2010, p. 43) “os agricultores que passam a utilizar esse pacote tecnológico (sementes transgênicas, adubos e venenos) serão sempre dependentes dessas empresas".

Em nível nacional, o movimento estudantil da agronomia realiza anualmente o seu principal fórum, o Congresso Nacional de Estudantes de Agronomia (CONEA), onde as temáticas mais importantes são discutidas pelos estudantes em mesas redondas já previamente programadas (VIEIRA; SOUZA; SOUZA, 2016). Ao final do congresso definem-se as deliberações, que são um conjunto de propostas que representam o posicionamento da militância acerca dos temas comumente abordados no evento. Em relação aos agrotóxicos, por exemplo, durante o $60^{\circ}$ CONEA, realizado em Santa Maria no estado do Rio Grande do Sul, deliberou-se (FEAB, 2017, p. 6):

O conjunto de militantes da Federação possui o compromisso de fortalecer e difundir a Campanha Permanente contra os Agrotóxicos e pela Vida, construindo espaços de discussão da campanha nas diferentes universidades, escolas estaduais e municipais do território nacional.

A denominada "campanha permanente contra os agrotóxicos e pela vida" é uma campanha organizada em nível nacional, que objetiva denunciar os efeitos dos agrotóxicos e do agronegócio para a população, além de anunciar alternativas para a produção de alimentos, Revista Extensão em Foco, nº 17, Out./ Dez. (2018), p. 40 - 53. 
especialmente os sistemas produtivos baseados em práticas sustentáveis e na Agroecologia (TYGEL et al., 2014).

Além da leitura e discussão sistemática de referências que abordam o tema, os estudantes militantes da FEAB organizam cinedebates, que são espaços onde discute-se o conteúdo cinematográfico de documentários previamente selecionados que abordam a temática. Os documentários "O veneno está na mesa" e "O veneno está na mesa 2" de Silvio Tendler são comumente abordados nesses cinedebates, sendo essa uma temática despertava grande interesse por parte dos estudantes.

Observa-se assim, que a federação pode representar uma importante ferramenta formadora de opinião no segmento estudantil, abordando diversas obras em seus espaços de discussão. Contudo, há outras obras importantes que poderiam ser debatidas, como propõe Lopes (2017), onde afirma que assim como é bom assistir aos filmes "O veneno está na mesa" e "O veneno está na mesa 2", de Silvio Tendler, seria bom também a leitura e discussão de outras obras, como a do livro "Agradeça aos agrotóxicos por estar vivo", de Nicholas Vital. Essa obra faz um contraponto as principais críticas empregadas ao uso de químicos na agricultura, demonstrando a importância do uso de agrotóxicos para ao aumento da produção agrícola e sua importância para o combate da fome no mundo (VITAL, 2017).

\section{CONCLUSÕES}

O uso de agrotóxicos e transgênicos na agricultura estão relacionados a diferentes problemas de ordem socioeconômica e ambiental. Os profissionais da agronomia foram os principais difusores dessas tecnologias para o campo, e sofreram diversas críticas de diferentes setores da sociedade. O movimento estudantil da agronomia representa um dos principais setores que conduzem uma crítica aos riscos dos agrotóxicos e as incertezas do uso dos transgênicos na agricultura e empreende diversas iniciativas para corroborar com a discussão dessa temática.

Em nível local, cada grupo do movimento estudantil promove encontros presenciais periodicamente, realizando-se a leitura e discussão de um conjunto de referenciais teóricos costumeiramente abordado pela FEAB. Também são realizados cinedebates e a confecção de cartilhas de divulgação que abordem a temática dos agrotóxicos e transgênicos. Contudo,

Revista Extensão em Foco, nº 17, Out./ Dez. (2018), p. 40 - 53. 
indicamos a possibilidade de ampliação dos referenciais teóricos adotados pelo movimento estudantil da agronomia. As iniciativas empreendidas pelo movimento estudantil nacional da agronomia corroboram para o fomento de uma crítica eminentemente popular acerca do uso dos agrotóxicos e dos transgênicos na agricultura.

\section{REFERÊNCIAS BIBLIOGRÁFICAS}

AMULEN, Deborah Ruth. et al. Environmental contaminants of honeybee products in Uganda detected using LC-MS/MS and GC-ECD. PLOS ONE, v. 12, n. 6, p. 1-14, 2017. Disponível em: 〈http://journals.plos.org/plosone/article?id=10.1371/journal.pone.0178546>. Acesso em Abril 2018.

ALMEIDA, Vicente Eduardo Soares de. et al. Uso de sementes geneticamente modificadas e agrotóxicos no Brasil: cultivando perigos. Ciência \& Saúde Coletiva, v. 22, n. 10, p. 3333 3339, 2017. Disponível em: < http://www.scielo.br/pdf/csc/v22n10/pt_1413-8123-csc-22-103333.pdf>. Acesso em Abril 2018.

ANDRIOLI, Antônio Inácio; FUCHS, Richard. Transgênicos: as sementes do mal. A silenciosa contaminação de solos e alimentos. $2^{\mathrm{a}}$ ed. São Paulo: Editora Expressão Popular, 2012. 288 p.

BARROWS, Geoffrey; SEXTON, Steven; ZILBERMAN, David. Agricultural biotechnology: the promise and prospects of genetically modified crops. Journal of Economic Perspectives, v. 28, n. $1, \quad$ p. 99-120, 2014. Disponível em: <https://www.aeaweb.org/articles?id=10.1257/jep.28.1.99>. Acesso em Abril 2018.

BELIK, Walter; CORREA, Vivian Helena Capacle. A crise dos alimentos e os agravantes para a fome mundial. Mundo Agrario, v. 14, n. 27, p. 1-28, 2013. Disponível em: $<$ http://www.mundoagrario.unlp.edu.ar/article/view/MAv14n27a01/pdf_1>. Acesso em Abril 2018.

BONNY, Sylvie. Genetically modified herbicide-tolerant crops, weeds, and herbicides: overview and impact. Environmental Management, v. 57, n. 1, p. 31-48, 2016. Disponível em: 〈https://link.springer.com/article/10.1007/s00267-015-0589-7>. Acesso em Abril 2018.

BURNS, Carol J.; PASTOOR, Timothy P. Pyrethroid epidemiology: a quality-based review. Critical Reviews in Toxicology, v. 48, n. 4, p. 297-311, 2017. Disponível em: $<$ https://www.tandfonline.com/doi/abs/10.1080/10408444.2017.1423463>. Acesso em Abril 2018.

CHABOUSOOU, Francis. Plantas doentes pelo uso de agrotóxicos: novas bases de uma prevenção contra doenças e parasitas. A teoria da trofobiose. São Paulo: Editora Expressão Popular, 2006. 320 p.

Revista Extensão em Foco, nº 17, Out./ Dez. (2018), p. 40 - 53. 
DEMARCHI, Layon Oreste. et al. Discutindo a agroecologia na universidade: grupo de extensão em agroecologia "Gira-Sol". Cadernos de Agroecologia, v. 6, n. 2, p. 1-5, 2011. Disponível em: < http://revistas.aba-agroecologia.org.br/index.php/cad/article/view/11391>. Acesso em Abril 2018.

FEDERAÇÃO DE ESTUDANTES DE AGRONOMIA DO BRASIL, FEAB. A formação profissional do engenheiro agrônomo no âmbito da agricultura familiar. In: $57^{\circ} \mathrm{CONEA}$, Curitiba/FEAB, 2014.

FEDERAÇÃO DE ESTUDANTES DE AGRONOMIA DO BRASIL, FEAB. Uma outra agricultura é possível. Qual a agronomia é necessária? In: $60^{\circ} \mathrm{CONEA}$, Santa Maria/FEAB, 2017.

FEDERAÇÃO DOS ESTUDANTES DE AGRONOMIA DO BRASIL, FEAB. O livro cinza do agronegócio. Curitiba: FEAB, 2010. Disponível em: < https://feab.files.wordpress.com/2008/08/2010-livrocinza-121220152015-phpapp02.pdf>.

Acesso em Abril 2018.

FISCHER, Klara. et al. Social impacts of GM crops in agriculture: systematic literature review. Sustainability, v. 7, n. 7, p. 8598-8620, 2015. Disponível em: < http://www.mdpi.com/2071-1050/7/7/8598>. Acesso em Abril 2018.

FONTOURA, Luiz Fernando Mazzini. A modernização do campo e o processo de deslocamento da cidade: Lavras do Sul - município da região da Campanha gaúcha. Geografia (Londrina), $\quad$ v. 25, n. 2, p. 19-39, 2016. Disponível em: < http://www.uel.br/revistas/uel/index.php/geografia/article/view/27369>. Acesso em Abril 2018.

FREIRE, Paulo. Extensão ou comunicação? $7^{\text {a }}$ ed. Rio de Janeiro: Paz e Terra, 1983. 93 p.

GOMES, Marco Antônio Ferreira; BARIZON, Robson Rolland Monticelli. Panorama da contaminação ambiental por agrotóxicos e nitrato de origem agrícola no Brasil: cenário 1992/2011. Jaguariúna: Embrapa Meio Ambiente, 2014. 35 p. Disponível em: < https://www.infoteca.cnptia.embrapa.br/bitstream/doc/987245/1/Doc98.pdf>. Acesso em Abril 2018.

HARARI, Yuval Noah. Sapiens. Uma breve história da humanidade. $9^{\mathrm{a}}$ ed. Porto Alegre: L\&PM, 2016. 464 p.

KHOSHNOOD, Zahra. Effects of environmental pollution on fish: a short review. Transylvanian Review of Systematical and Ecological Research, v. 19, n. 1, p. 49-60, 2017. Disponível em: <https://www.degruyter.com/view/j/trser.2017.19.issue-1/trser-20170005/trser-2017-0005.xml>. Acesso em Abril 2018.

Revista Extensão em Foco, nº 17, Out./ Dez. (2018), p. 40 - 53. 
KLÜMPER, Wilhelm; QAIM, Matin. A meta-analysis of the impacts of genetically modified crops. PLOS ONE, v. 9, n. 11, 2014. Disponível em: <https://doi.org/10.1371/journal.pone.0111629>. Acesso em Abril 2018.

KNICKEL, Karlheinz. et al. Agricultural modernization and sustainable agriculture: contradictions and complementarities. International Journal of Agricultural Sustainability, v. $17, \quad$ n. $5, \quad$ p. 1-18, 2017. Disponível em: <https://www.tandfonline.com/doi/abs/10.1080/14735903.2017.1373464>. Acesso em Abril 2018.

LABORDE, David Debucquet; MARTIN, Will. Implications of slowing growth in emerging market economies for hunger and poverty in rural areas of developing countries. Washington, D.C.: International Food Policy Research Institute, 2016. 56 p. Disponível em: < http://www.ifpri.org/publication/implications-slowing-growth-emerging-market-economieshunger-and-poverty-rural-areas>. Acesso em Abril 2018.

LI, Huizhen. et al. Global occurrence of pyrethroid insecticides in sediment and the associated toxicological effects on benthic invertebrates: an overview. Journal of Hazardous Materials, v. 324, p. 258-271, 2017. Disponível em: <https://www.sciencedirect.com/science/article/pii/S0304389416309785?via\%3Dihub>.

Acesso em Abril 2018.

LIMA, Maria Cecília de; ROCHA, Sá de Alencar. Efeitos dos agrotóxicos sobre as abelhas silvestres no Brasil: Proposta metodológica de acompanhamento. Goiás: Ibama, 2012. 88 p.

LLORCA, Marta. et al. Review of emerging contaminants in aquatic biota from Latin America: 2002-2016. Evironmental Toxycology and Chemistry, v. 36, n. 7, p. 1716-1727, 2017. Disponível em: 〈https://setac.onlinelibrary.wiley.com/doi/abs/10.1002/etc.3626〉. Acesso em Abril de 2018.

LOPES, Carlos. É possível produzir alimentos para o Brasil sem agrotóxicos? Revista Ciência e Cultura, v. $69, \quad$ n. $4, \quad$ p. 52-55, 2017. Disponível em: < http://cienciaecultura.bvs.br/scielo.php?script=sci_arttext\&pid=S0009-67252017000400016>. Acesso em Abril 2018.

MACHADO, Luiz Carlos Pinheiro; MACHADO FILHO, Luiz Carlos Pinheiro. A dialética da agroecologia: contribuição para um mundo com alimentos sem veneno. $1^{\mathrm{a}}$ ed. São Paulo: Editora Expressão Popular, 2014. 360 p.

MAZOYER; Marcel; ROUDART, Laurence. História das agriculturas no mundo: do neolítico à crise contemporânea. São Paulo: Editora UNESP; Brasília, DF: NEAD, 2010. 568 p.

Revista Extensão em Foco, nº 17, Out./ Dez. (2018), p. 40 - 53. 
MOTTA, Renata. Transnational discursive opportunities and social movement risk frames opposing GMOs. Social Movement Studies, v. 14, n. 5, p. 576-595, 2015. Disponível em: $<$ https://www.tandfonline.com/doi/full/10.1080/14742837.2014.947253>. Acesso em Abril 2018.

OTERO, Geraldo; LAPEGNA, Pablo. Transgenic crops in Latin America: expropriation, negative value and the state. Journal of Agrarian Change, v. 16, n. 4, p. 665-674, 2016. Disponível em: 〈https://onlinelibrary.wiley.com/doi/pdf/10.1111/joac.12159> . Acesso em Abril de 2018.

PINHEIRO, Sebastião; NASR, Nasser Youssef; LUZ, Dioclécio. A agricultura ecológica e a máfia dos agrotóxicos no Brasil. Porto Alegre: Fundação Juquira Candirú, 1993. p. 338.

PINTOR, Eduardo de; SILVA, Geisiane Michelle de; PIACENTI, Carlos Alberto. Crédito rural e crescimento econômico no Brasil. Revista Política Agrícola, v. 24, n. 1, p. 5-19, 2015. Disponível em: <https://seer.sede.embrapa.br/index.php/RPA/article/view/962/852>. Acesso em Abril 2018.

PORTO, Marcelo Firpo; SOARES, Wagner Lopes. Modelo de desenvolvimento, agrotóxicos e saúde: um panorama da realidade agrícola brasileira e propostas para uma agenda de pesquisa inovadora. Revista Brasileira de Saúde Ocupacional, v. 27, n. 125, p. 17-50, 2012. Disponível em: < http://www.scielo.br/pdf/rbso/v37n125/a04v37n125.pdf>. Acesso em Abril 2018.

RAMOS, Rodrigo Ferraz. et al. Agroecologia e extensão: o movimento estudantil em defesa de uma nova agronomia. Revista Brasileira de Extensão Universitária, v. 8, n. 3, p. 135-142, 2017a. Disponível em: < https://periodicos.uffs.edu.br/index.php/RBEU/article/view/4779>. Acesso em Abril 2018.

RAMOS, Rodrigo Ferraz. et al. Experiências didático-pedagógicas em agroecologia na Região Noroeste do Rio Grande do Sul. Revista Brasileira de Extensão Universitária, v. 8, n. 1, p. 15-22, 2017b. Disponível em: < https://periodicos.uffs.edu.br/index.php/RBEU/article/view/4762>. Acesso em Abril 2018.

RIBEIRO, Isabelle Geoffroy; MARIN, Victor Augustus. A falta de informação sobre os organismos geneticamente modificados no Brasil. Ciência \& Saúde Coletiva, v. 17, n. 02, p. 359-368, 2012. Disponível em: < http://www.scielo.br/pdf/csc/v17n2/a10v17n2.pdf>. Acesso em Abril 2018.

SANTANA, Vilma Souza; MOURA, Maria Claudia Peres; NOGUEIRA, Flávia Ferreira. Occupational pesticide poisoning, 2000-2009, Brazil. Revista Saúde Pública, v. 47, n. 03, p. 1-8, 2013. Disponível em: < http://www.scielo.br/pdf/rsp/v47n3/en_0034-8910-rsp-47-030598.pdf>. Acesso em Abril 2018.

SIMTION, Daniela. The position of agriculture, agro-food system and national economy. Scientific Papers Series Management, Economic Engineering in Agriculture and Rural Revista Extensão em Foco, nº 17, Out./ Dez. (2018), p. 40 - 53. 
Development, v. 17, n. 1, p. 413-418, 2017. Disponível $<$ http://managementjournal.usamv.ro/index.php/scientific-papers/1378-the-position-ofagriculture-agro-food-system-and-national-economy-1378> . Acesso em Abril 2018.

em:

SILVA, Luiz Mauro Santos. A abordagem sistêmica na formação do agrônomo do século XXI. $1^{\text {a }}$ ed. Curitiba: Editora Appris, 2011.

SILVA NETO, Benedito. A agronomia e o desenvolvimento sustentável: por uma ciência da complexidade. Desenvolvimento em questão. ano. 7, n. 13, p. 37-62, 2009. Disponível em: < https://www.revistas.unijui.edu.br/index.php/desenvolvimentoemquestao/article/view/166>.

Acesso em Abril 2018.

SILVA NETO, Benedito. A questão agroecológica: uma perspectiva ecossocialista. $1^{\mathrm{a}}$ ed. Curitiba: CRV, 2017. v. 1. 236 p.

SILVA NETO, Benedito; BASSO, David. Sistemas agrários do Rio Grande do Sul: Análise e Recomendações de Políticas. 2ª ed. Ijuí: Editora UNIJUÍ, 2015. 336 p.

STANDAGE, Tom. Uma história comestível da humanidade. São Paulo: Editora Zahar, 2010. 216 p.

TYGEL, Alan Freihof, et al. Campanha permanente contra os agrotóxicos e pela vida: construção da resistência brasileira ao avanço do capital no campo. Agriculturas, v. 11, n. 4, p. 38-42, 2014. Disponível em: <http://aspta.org.br/revista/v11-n4-alimentacao-adequada-esaudavel/campanha-permanente-contra-os-agrotoxicos-e-pela-vida-construcao-da-resistenciabrasileira-ao-avanco-do-capital-no-campo/>. Acesso em Abril 2018.

VIEIRA, Lucas Moretz-Sohn David; SOUZA, Paulo Marcelo; SOUZA, Hadma Milaneze de. Da trajetória no movimento estudantil da agronomia à formação de uma(a) profissional comprometido com a questão social e ambiental do meio rural. In: Anais do $54^{\circ}$ SOBER, UFAL, 2016. Disponível em: 〈http://icongresso.itarget.com.br/tra/arquivos/ser.6/1/7097.pdf>. Acesso em Abril 2018.

VITAL, Nicholas. Agradeça aos agrotóxicos por estar vivo. Rio de Janeiro: Editora Record LTDA, 2017. 252 p.

ZANONI, Magda; FERMENT, Gilles. Transgênicos para quem? Agricultura, ciência e sociedade. Brasília: MDA, 2011. 520 p. Disponível em: <http://aspta.org.br/wpcontent/uploads/2011/06/Transgenicos_para_quem.pdf>. Acesso em Abril 2018.

Revista Extensão em Foco, nº 17, Out./ Dez. (2018), p. 40 - 53. 